\title{
The ABC Matrix
}

\section{Ernesto Estrada}

the date of receipt and acceptance should be inserted later

\begin{abstract}
The atom-bond connectivity index-abbreviated $A B C$ - has proven to describe the heat of formation of alkanes with an accuracy comparable to that of high-level $a b$ initio and DFT methods. Its physical interpretation in terms of quantumtheory, its generalization, and many of its mathematical properties have been reported. Here, a probabilistic interpretation of the generalized $A B C$ index is provided. It indicates that the terms defining this index represent the probability of visiting a nearest neighbor edge from one side or the other of a given edge in a graph. This interpretation is general enough as to cover the case of polarizing bonds in a molecular context. We then introduce a matrix representation of these probabilities in the form of generalized $A B C$ matrices. Using this representation we study some indices related to the eigenvalues of the $A B C$ matrices, such as the $A B C$ energy and the $A B C$ Estrada index. We provide some bounds for these parameters in general graphs.
\end{abstract}

Keywords ABC index, Graph energy, Estrada index, spectral theory, probabilistic interpretation

\section{Introduction}

The history of topological indices - defined as numerical values associated with chemical constitution for correlation of chemical structure with various physical properties, chemical reactivity or biological activity (Van de Waterbeemd et al. (1997)) — dates back to the pioneering efforts of using graph theory to study molecular problems (Balaban and Ivanciuc (2000)). A relatively late arrival to the wide list of topological molecular descriptors was the "atom-bond connectivity index" developed by Estrada et al. (1998). This index, appropriately abbreviated by $A B C$ is defined by

$$
A B C(G)=\sum_{(i, j) \in E} \sqrt{\frac{k_{i}+k_{j}-2}{k_{i} k_{j}}},
$$

where $k_{i}$ is the degree of the node $i$ - the number of edges incident to that node in the graph $G=(V, E)$.

The $A B C$ index was shown to have a good correlation with the heat of formation $\Delta H_{f}^{\circ}$ of alkanes (Estrada et al. (1998)). In a subsequent critical evaluation of this descriptive capacity of the $A B C$ index for the heat of formation of alkanes Gutman et al. (2012b) confirmed that it "reproduces the heat of formation with an accuracy comparable to that of high-level ab initio and DFT (MP2, B3LYP) quantum chemical calculations". Estrada (2008) provides a quantum-chemical explanation for this descriptive property of the $A B C$ index based on the ratio of 1,3 -interactions with respect to the total number of $1,2-, 1,3-$ and 1,4-interactions in alkanes. It was concluded that the heat of formation of alkanes can be obtained as a combination of stabilizing effects coming from atoms, bonds and protobranches. This has prompted Gutman (2013) to claim that the "ABC happens to be the only topological index for which a theoretical, quantum-theory-based, foundation and justification has been found." This index also triggers a large series of papers in mathematics (Ahmadi et al. (2013b,a); Chen and Guo (2011); Chen et al. (2012); Das et al. (2011); Gutman and Furtula (2012); Gutman et al. (2012a); Furtula et al. (2012); Lin et al. (2013); Vassilev and Huntington (2012); Fath-Tabar et al. (2011); Xing et al. (2011)), specially due to the difficulties found to identify the graphs with minimal $A B C$ index, which has been coined as the " $A B C$ index conundrum" (Gutman et al. (2013)).

As a collateral effect of this interest in the high correlation abilities of the $A B C$ index for the heat of formation of alkanes, Furtula et al. (2010) make a generalization of this index to consider

$$
A B C_{\gamma}(G)=\sum_{(i, j) \in E}\left(\frac{k_{i}+k_{j}-2}{k_{i} k_{j}}\right)^{\gamma}
$$

Department of Mathematics \& Statistics, University of Strathclyde, 26 Richmond Street, Glasgow G11HQ, UK 
where they showed that when $\gamma=-3$ even better correlation is obtained with the $\Delta H_{f}^{\circ}$ of alkanes. As Gutman (2013) has stressed this index was "somewhat inadequately named "augmented Zagreb index"". We will call it hereafter the generalized ABC index.

It is obvious from these previous observations that the descriptive capacity of the $A B C$ indices resides very much in the term $\left(k_{i}+k_{j}-2\right) /\left(k_{i} k_{j}\right)$. Our main goal here is then to further investigate the topological nature of this ratio in the most general possible way. Although a quantum-chemical explanation of the capacity of $A B C$-like indices was previously provided (Estrada (2008)), the current investigation will provide a probabilistic explanation that fits very well with the chemical intuition for understanding the capacity of these indices to describe the energetics of alkanes. Furthermore, it will also allow further investigations of more general scenarios outside molecular sciences, such as the study of random walks on graphs. We then formulate a matrix representation of the generalized $A B C$ indices and study some indices derived from it, which hopefully will open new avenues in the study of the $A B C$-like analysis of (molecular) graphs.

\section{A probabilistic framework for $A B C$ indices}

We start by considering a molecular graph $G=(V, E)$ which is a simple, connected graph with $n$ nodes representing the atoms of the molecule, excluding any hydrogen, and $m$ edges representing the covalent bonds among the $n$ atoms. The theory presented here is however of application to any connected, simple graph as we will see later on in this work. The degree of a node, represented here by $k_{i}$, is the number of nodes adjacent to it. In a chemical context it represents the valency of the corresponding atom in the hydrogen-depleted graph. Let us consider two adjacent atoms $i$ and $j$ in the molecule and let us consider that there are two electrons $\tilde{i}$ and $\tilde{j}$ that belong to the atoms $i$ and $j$, respectively. Then, we ask the following question: What is the probability that the electron $\tilde{i}$ 'polarizes' one of the bonds incident to the atom $i$ OR the electron $\tilde{j}$ polarizes one of the bonds incident to the atom $j$ ? By 'polarize' here we mean the following. Suppose that the electron $\tilde{i}$ on the atom $i$ is displaced to the nearest neighbor atom $r$, then the atom $i$ will have a positive electron charge density and the atom $r$ will have a negative one. Thus the bond $(i, r) \in E$ is polarized. Now, we also want to consider here the situation in which both electrons are located at their respective atoms. Then, the total number of scenarios here are as follow. Let $(i, j) \in E$ be the polarizing bond. Then, it can polarize any of its nearest neighbors, which total $k\left(e_{i j}\right)=k_{i}+k_{j}-2$. Because we also want to count the possibility that both electrons remain located at their respective atoms we have $S_{i j}=k\left(e_{i j}\right)+1=k_{i}+k_{j}-1$ single-bond polarizing scenarios. In addition, we want to exclude the double polarizing scenarios. That is, such cases in which the electron $\tilde{i}$ polarizes any of the bonds incident to the atom $i$ AND the electron $\tilde{j}$ polarizes one of the bonds incident to the atom $j$. Excluding the self-polarization of the bond $(i, j) \in E$ we have that these double polarization scenarios sum $D_{i j}=\left(k_{i}-1\right)\left(k_{j}-1\right)$. Thus, the probability that the electron $\tilde{i}$ 'polarizes' one of the bonds incident to the atom $i$ OR the electron $\tilde{j}$ polarizes one of the bonds incident to the atom $j$ is given by

$$
\begin{aligned}
\tilde{P}_{i j} & =\frac{k\left(e_{i j}\right)}{S_{i j}+D_{i j}} \\
& =\frac{k_{i}+k_{j}-2}{k_{i}+k_{j}-1+\left(k_{i}-1\right)\left(k_{j}-1\right)} \\
& =\frac{k_{i}+k_{j}-2}{k_{i} k_{j}}
\end{aligned}
$$

The probability of simultaneously polarizing two bonds in the form described previously is $\tilde{D}_{i j}=\frac{\left(k_{i}-1\right)\left(k_{j}-1\right)}{k_{i} k_{j}}$, and the probability of both electrons remaining at the bond is $\tilde{R}_{i j}=\frac{1}{k_{i} k_{j}}$. Obviously, $\tilde{P}_{i j}+\tilde{D}_{i j}+\tilde{R}_{i j}=1$ as required for probabilities. An illustrative example is provided in Figure (2.1) where we give all the possibilities of polarization of bonds incident with the atoms $i$ and $j$ in a graph which can be representing the molecule of 2,2,3-trimethylbutane. The first image corresponds to the non-polarization of bonds, from the second to the sixth they correspond to the polarization of one bond, and the rest correspond to the simultaneous polarization of two bonds. 

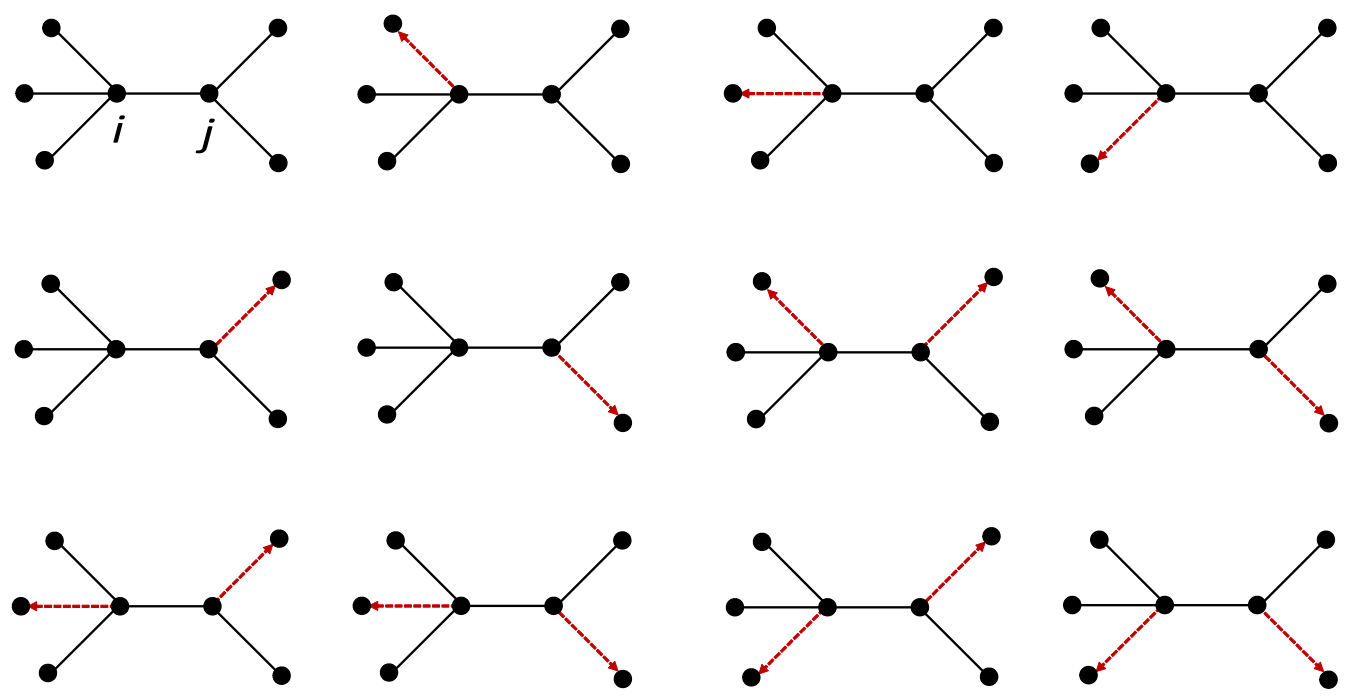

Fig. 2.1 Illustration of the bond polarization scenarios for the hydrogen-depleted graph representing 2,2,3-trimethylbutane. Red broken line represents the polarized bond, where the head of the arrow points to the atoms with negative electron density and the tail points to the one with a positive electron charge density. The first structure represents the case where the two electrons remain at the corresponding atoms.

The reason for which we exclude the possibility of polarizing two bonds at the same time is because of the obvious chemical fact that such polarization will make the bond $(i, j) \in E$ very unstable due to the presence of two positive charges on the adjacent atoms of this bond. It is important to remark that $P_{i j}$ indicates the polarizing capacity of the bond $(i, j) \in E$ and not its capacity of being polarized by other bonds. For instance, in 2,2,3-trimethylbutane we have: $P_{C H_{3}}-C=3 / 4, P_{C-C H}=5 / 12$, and $\mathrm{P}_{\mathrm{CH}-\mathrm{CH}_{3}}=2 / 3$. Thus, the central bond $\mathrm{C}-\mathrm{CH}$ has the least polarizing capacity. However, it is surrounded by highly polarizing bonds, which means that — on a purely probabilistic way - it can be easily polarized. It is a well-known fact that such central bonds in alkanes are more elongated than the peripheral one, which agrees very well with the intuition presented in this paper.

How is the polarizing capacity reflected on the stabilization of the corresponding molecule? The polarizing capacity of a given bond indicates its capacity to delocalize the electrons in that bond through nearest neighbor bonds. Such electron delocalization is thought to decrease the heat of formation of the molecule and being responsible for the good correlations between $A B C$-like indices and the heat of formation of alkanes.

\section{Definition of the ABC matrix and related indices}

We now start with the definition of the $A B C$ matrix. First, let us consider the Hamiltonian of the corresponding molecule in a similar way in which the adjacency matrix is used in the tight-binding (Hückel molecular orbital, HMO) method (Kutzelnigg (2007)). This Hamiltonian is then written as

$$
\hat{H}_{\gamma}=\tilde{\alpha} I+\tilde{\beta} M_{\gamma}
$$

where $\alpha=0$ and $\beta=-1$ are the typical HMO parameters and the entries of the $\hat{M}_{\gamma}$ are given in terms of the kinetic $T_{i j}$ and potential $V_{i j}$ energies of the bond

$$
\begin{aligned}
\hat{M}_{\gamma}(i, j) & =\left(T_{i j}-V_{i j}\right)^{\gamma} \\
& =\left[\left(\frac{1}{k_{i}}+\frac{1}{k_{j}}\right)-\frac{2}{k_{i} k_{j}}\right]^{\gamma} .
\end{aligned}
$$

Notice that we consider the kinetic energy of the bond on the basis of assuming that each electron is a random walker that has probability of jumping to a nearest neighbor given by $\frac{1}{k_{i}}$ (respectively $\frac{1}{k_{k}}$ ) (Lovász (1993)). The potential energy is given by the interaction of both electrons given by the sum of the number of scenarios of simultaneous polarization of two bonds plus the scenario in which both electrons remain in the corresponding bond. Let us then call $\hat{H}_{\gamma}$ the polarization-HMO Hamiltonian. We then have the following definitions of terms that we will use in this work.

Definition 1 The $A B C$ matrix is the matrix $M_{\gamma} \in \mathbb{R}^{n \times n}$ whose entries are given as follows:

$$
M_{\gamma}(i, j)=\left\{\begin{array}{cc}
P_{i j}^{\gamma} & (i, j) \in E \\
0 & (i, j) \notin E,
\end{array}\right.
$$


Let $K$ be the diagonal matrix whose nonzero entries are the degrees of the nodes and let $A$ be the adjacency matrix of the corresponding graph. Then, the $A B C$ matrix can be obtained as follow

$$
\begin{aligned}
M_{\gamma} & =\left(K^{-1}[(K-I) A+A(K-I)] K^{-1}\right)^{\circ \gamma} \\
& =\left(A K^{-1}+K^{-1} A-2 K^{-1} A K^{-1}\right)^{\circ \gamma},
\end{aligned}
$$

where $\circ$ indicates the entrywise - also known as Hadamard or Schur-operation (Horn and Johnson (2012)). Notice that the matrices $A K^{-1}$ and $K^{-1} A$ are the transition probability matrices for random walkers on the graph (Lovász (1993)).

Definition 2 The generalized Randić index (Randić (1975); Li and Shi (2008)) is defined as

$$
\chi_{\gamma}=\sum_{(i, j) \in E}\left(k_{i} k_{j}\right)^{\gamma} .
$$

Definition 3 The energy levels of a molecule in the polarization-HMO method are defined as the negative of the eigenvalues of $M_{\gamma}$. That is, $E_{\gamma, j}=-\tilde{\lambda}_{\gamma, j}$, where $\tilde{\lambda}_{\gamma j}$ is the $j$ th eigenvalue of $M_{\gamma}$.

Definition 4 The graph-theoretic energy - for a compendium about graph energy see (Li et al. (2012)) —of the molecule in the polarization-HMO method is defined as $E_{\gamma}=\sum_{j=1}^{n}\left|\tilde{\lambda}_{\gamma j}\right|$. When $\gamma=1 / 2$ we will call this the ABC total energy of the graph, $E_{A B C}$.

Definition 5 The electronic partition function of the molecule in the polarization-HMO method is defined as

$$
Z_{\gamma}=\sum_{j=1}^{n} \exp \left(\beta \tilde{\lambda}_{\gamma j}\right),
$$

where $\tilde{\beta}$ is the inverse temperature of the system. This is known as the Estrada index of the graph (Estrada (2000); Estrada and Rodriguez-Velazquez (2005); Estrada and Hatano (2007)) and typically it is studied for $\tilde{\beta} \equiv 1$ (for review about the Estrada index of graphs see (Deng et al. (2009); Gutman et al. (2011))). When $\gamma=1 / 2$ we will call this the $A B C$ Estrada index of the graph, $E E_{A B C}$. Another interpretation of the Estrada index has been provided in the context of thermal Green's functions of quantum harmonic oscillators (Estrada et al. (2012); Estrada (2012)). For the general theory about the use of matrix functions in graph theory see Estrada and Higham (2010).

\section{Mathematical results}

4.1 Spectral radius and total energies

Let $\tilde{\lambda}_{\gamma, j}=\tilde{\lambda}_{j}\left(M_{\gamma}\right)$ and let us consider the eigenvalues of $M_{\gamma}$ ordered in nonincreasing order as: $\tilde{\lambda}_{\gamma, 1}>\tilde{\lambda}_{\gamma, 2} \geq \cdots \geq \tilde{\lambda}_{\gamma, n}$. The largest eigenvalue $\tilde{\lambda}_{\gamma, 1}$ is known as the spectral radius of the corresponding matrix (Horn and Johnson (2012)). Let $\boldsymbol{\psi}_{\gamma, j}$ be the eigenvector associated with the eigenvalue $\tilde{\lambda}_{\gamma, j}$. The first obvious result is that the $A B C$ index can be expressed as a quadratic form of the $A B C$ matrix. That is,

$$
A B C_{\gamma}=\frac{1}{2}\left(\mathbf{1}^{T} M_{\gamma} \mathbf{1}\right)
$$

where $\mathbf{1}$ is an all-ones vector. Then, let

$$
M_{\gamma}=V_{\gamma} \tilde{\Lambda_{\gamma}} V_{\gamma}^{T}
$$

be the spectral decomposition of the $A B C$ matrix, where $V_{\gamma}=\left[\boldsymbol{\psi}_{\gamma, 1} \cdots \boldsymbol{\psi}_{\gamma, n}\right]$ and $\tilde{\Lambda}_{\gamma}$ is the diagonal matrix of eigenvalues of $M_{\gamma}$. It is straightforward to realize that

$$
A B C_{\gamma}=n \sum_{j=1}^{n} \tilde{\lambda}_{\gamma, j} \cos ^{2} \theta_{\gamma, j},
$$

where $\theta_{\gamma, j}$ is the angle between the corresponding orthonormalized eigenvector $\boldsymbol{\psi}_{\gamma, j}$ and the vector 1 . Then, when the spectral gap of the $A B C$ matrix is very large, i.e., $\tilde{\lambda}_{\gamma, 1} \gg \tilde{\lambda}_{\gamma, 2}$, the $A B C_{\gamma}$ index can be approximated by 


$$
A B C_{\gamma} \approx n \tilde{\lambda}_{\gamma, 1} \cos ^{2} \theta_{\gamma, 1} .
$$

We will now obtain some bounds for the spectral radius of the $A B C$ matrix. Let $A B C_{\gamma}(i)$ be the sum of the $i$ th row or column of the $A B C$ matrix. That is, $A B C_{\gamma}=\frac{1}{2} \sum_{i} A B C_{\gamma}(i)$. Then, the following result is a simple application of standard linear algebra theory.

Lemma 1 The spectral radius of the ABC matrix is bounded as

$$
\frac{2}{n} A B C_{\gamma} \leq \tilde{\lambda}_{\gamma, 1} \leq \max _{i} A B C_{\gamma}(i),
$$

with equality at both sides if and only if $A B C_{\gamma}(i)$ is the same for every node $i$ of the graph.

Lemma 2 Let $\gamma=\frac{1}{2}$ and let $\tilde{\lambda}_{1}\left(M_{\gamma}\right)$ be the largest eigenvalue of the matrix $M_{\gamma}$. Then,

$$
\tilde{\lambda}_{1}\left(M_{\frac{1}{2}}\right) \leq \sqrt{\lambda_{1}(B) \cdot \lambda_{1}\left(R_{1}\right)} .
$$

Proof Let

$$
B_{\gamma}=(K A+A K-2 A)^{\circ \gamma}
$$

and

$$
R_{\gamma}=\left(K^{-1} A K^{-1}\right)^{\circ \gamma}
$$

which is the so-called Randić matrix (Bozkurt et al. (2010)) when $\gamma=1 / 2$. Then, we can express the $A B C$ matrix as follows

$$
M_{\gamma}=B_{\gamma} \circ R_{\gamma}
$$

It is known that if $P, Q \in \mathbb{R}^{n \times n}$ are nonnegative and $\gamma \in[0,1]$ then

$$
\lambda_{1}\left(P^{\circ \gamma} \circ Q^{\circ(1-\gamma)}\right) \leq \sqrt{\lambda_{1}(P) \cdot \lambda_{1}(Q)} .
$$

Then, the result follows for the case $\gamma=\frac{1}{2}$.

The previous result is specific for the case $\gamma=\frac{1}{2}$ because it is the only case in which the exponent of $P$ and $Q$ in 4.9 is the same. For other values of $\gamma$ other techniques must be used to bound the spectral radius of $M_{\gamma}$.

Now we prove a result that connects the $A B C$ total energy with the general Randić index $\chi_{-1}$.

Lemma 3 Let $\gamma=\frac{1}{2}$, then the $A B C$ energy of a graph is bounded as

$$
E_{A B C} \leq \sqrt{2 n^{2}-4 n \chi-1} \leq \sqrt{2 n^{2}-4 E_{R}^{2}},
$$

where $E_{R}$ is the Randić energy of the graph (Bozkurt et al. (2010)).

Proof We start by considering the second moment of the $A B C$ matrix,

$$
\begin{aligned}
\operatorname{tr}\left(M_{1 / 2}\right)^{2} & =2 \sum_{(i j) \in E} P_{i j}^{2}=2 \sum_{(i j) \in E} \frac{k_{i}+k_{j}-2}{k_{i} k_{j}} \\
& =2 n-4 \chi_{-1}
\end{aligned}
$$

Now using the fact that the variance of the absolute value of a series of numbers is nonnegative we have

$$
\frac{1}{n} \sum_{j}\left|\tilde{\lambda}_{j}\right|^{2}-\left(\frac{1}{n} \sum_{j}\left|\tilde{\lambda}_{j}\right|\right)^{2} \geq 0
$$

which implies that

$$
\frac{1}{n} \operatorname{tr}\left(M_{1 / 2}\right)^{2}-\frac{1}{n^{2}} E_{A B C}^{2} \geq 0
$$


Then, we have that

$$
\begin{aligned}
E_{A B C} & \leq \sqrt{n t r\left(M_{1 / 2}\right)^{2}}, \\
& =\sqrt{2 n^{2}-4 n \chi-1} .
\end{aligned}
$$

Finally we use the known inequality (Bozkurt et al. (2010))

$$
E_{R} \leq \sqrt{2 n \chi-1}
$$

to prove the last inequality.

\subsection{ABC Estrada index}

This subsection is devoted to the analysis of the $A B C$ Estrada index of graphs. We start by proving some upper bounds for this index in general connected graphs.

Lemma 4 Let $\gamma=\frac{1}{2}$, then the ABC Estrada index of a simple connected graph is bounded as

$$
E E_{A B C} \leq n-1+e^{\sqrt{2 n-4 \chi-1-1}}
$$

Proof Let $n_{+}$be the number of positive eigenvalues of the $A B C$ matrix, then we can write the $A B C$ index as

$$
\begin{aligned}
E E_{A B C} & =\sum_{j=1}^{n} e^{\tilde{\lambda}_{j}} \leq n-n_{+}+\sum_{j=1}^{n_{+}} e^{\tilde{\lambda}_{j}} \\
& =n-n_{+}+\sum_{i=1}^{n_{+}} \sum_{k \geq 0}^{\infty} \frac{\tilde{\lambda}_{j}^{k}}{k !} \\
& =n+\sum_{k \geq 1}^{\infty} \frac{1}{k !} \sum_{i=1}^{n_{+}}\left(\tilde{\lambda}_{j}^{2}\right)^{k / 2} \\
& \leq n+\sum_{k \geq 1}^{\infty} \frac{1}{k !}\left(\sum_{i=1}^{n_{+}} \tilde{\lambda}_{j}^{2}\right)^{k / 2} \\
& =n+\sum_{k \geq 1}^{\infty} \frac{1}{k !}\left(t r\left(M_{1 / 2}^{2}\right)-\sum_{i=n_{+}+1}^{n} \tilde{\lambda}_{j}^{2}\right)^{k / 2} \\
& =n+\sum_{k \geq 1}^{\infty} \frac{1}{k !}\left(2 n-4 \chi-1-\sum_{i=n_{+}+1}^{n} \tilde{\lambda}_{j}^{2}\right)^{k / 2}
\end{aligned}
$$

If the graph contains at least one edge, i.e., it has $K_{2}$ as an induced subgraph, then $\sum_{i=n_{+}+1}^{n} \tilde{\lambda}_{j}^{2} \geq 1$. This is due to the fact that $K_{2}$ has eigenvalues 1 and -1 and then from the interlacing theorem $\tilde{\lambda}_{n}(G) \leq-1$, which implies the previous inequality. Therefore,

$$
\begin{aligned}
E E_{A B C} & \leq n+\sum_{k \geq 1}^{\infty} \frac{1}{k !}\left(2 n-4 \chi_{-1}-1\right)^{k / 2} \\
& =n-1+e^{\sqrt{2 n-4 \chi-1}-1}
\end{aligned}
$$

as required.

Another upper bound for the $A B C$ Estrada index is provided in the following result, which shows that the among all graphs with $n$ nodes the maximum of this index is reached for the complete graph. 
Lemma 5 Let $G$ be a simple connected graph on $n$ nodes and let $\gamma=\frac{1}{2}$. Then, the ABC Estrada index of $G$ is bounded as follows

$$
E E_{A B C}(G) \leq e^{\sqrt{2(n-2)}}+(n-1) \exp \left(\frac{-\sqrt{2(n-2)}}{n-1}\right)
$$

with equality if and only if the graph is complete.

Proof Let $l$ be an edge of $G$ and assume that $G$ is not trivial, i.e., it contains at least one edge. Let $G-l$ be the graph resulting from removing the edge $l$ from $G$. Let $\mu_{k}(G)$ be the number of closed walks of length $k$ in $G$. Then, $\mu_{k}(G-l)=\mu_{k}(G)-\mu_{k}(G: l)$, where $\mu_{k}(G: l)$ is the number of closed walks of length $k$ in $G$ which contain the edge $l$. Consequently,

$$
\begin{gathered}
\sum_{p=1}^{n}\left(\sum_{k=0}^{\infty} \frac{\mu_{k}(G-l)}{k !}\right)_{p p} \leq \sum_{p=1}^{n}\left(\sum_{k=0}^{\infty} \frac{\mu_{k}(G)}{k !}\right)_{p p} \\
\operatorname{tr} \exp \left(M_{\gamma}(G-l)\right) \leq \operatorname{tr} \exp \left(M_{\gamma}(G)\right)
\end{gathered}
$$

which means that $E E_{A B C}(G) \leq E E_{A B C}\left(K_{n}\right)$ with equality if the graph is the complete graph with $n$ vertices. We now obtain the formula for $E E_{A B C}\left(K_{n}\right)$. The spectrum of the adjacency matrix of $K_{n}$ is $\lambda_{1}=n-1$ with multiplicity one and $\lambda_{j \geq 2}=-1$ with multiplicity $n-1$. The degree of every node in $K_{n}$ is $k_{i}=n-1$. Thus, $P_{i j}=\frac{\sqrt{2(n-2)}}{n-1}$, for every pair of nodes in the graph. Consequently, the eigenvalues of the $A B C$ matrix of $K_{n}$ are: $\lambda_{1}=\sqrt{2(n-2)}$ with multiplicity one and $\lambda_{j \geq 2}=-\frac{\sqrt{2(n-2)}}{n-1}$ with multiplicity $n-1$, from which the result finally comes.

Corollary 1 Let $G$ be a graph and let $T$ be an induced tree of $G$ and let $\gamma=\frac{1}{2}$. Then

$$
E E_{A B C}(G) \geq E E_{A B C}(T)
$$

We now move to prove that among all graphs with $n$ nodes the graph with the minimal $A B C$ Estrada index is the path graph. First, we obtain the expression for $E E_{A B C}\left(P_{n}\right)$.

Lemma 6 Let $P_{n}$ be a path with $n$ nodes and let $\gamma=\frac{1}{2}$. Then, when $n \rightarrow \infty$

$$
E E_{A B C}\left(P_{n}\right)=(n+1) I_{0}(\sqrt{2})
$$

Proof It is straightforward to realize that $M_{1 / 2}\left(P_{n}\right)=\frac{1}{\sqrt{2}} A\left(P_{n}\right)$. Then

$$
E E_{A B C}\left(P_{n}\right)=\sum_{j=1}^{n} e^{(2 / \sqrt{2}) \cos (\pi j /(n+1))} .
$$

Now, when $n \rightarrow \infty$ the summation in the previous expression can be evaluated by making use of the following integral

$$
I_{0}(x)=\frac{1}{\pi} \int_{0}^{\pi} \exp (x \cos \theta) d \theta
$$

where $I_{0}(x)$ is the corresponding modified Bessel function of the first kind. Then

$$
\begin{aligned}
\operatorname{EE}_{A B C}\left(P_{n}\right) & =(n+1) \int_{0}^{\pi} e^{(2 / \sqrt{2}) \cos (\pi j /(n+1))} \\
& =(n+1) I_{0}(\sqrt{2})
\end{aligned}
$$

where $\theta=\frac{j \pi}{n+1}$.

Now, we can find the lower bound for the Estrada $A B C$ index of graphs. 
Lemma 7 Let $\gamma=\frac{1}{2}$ and let $G$ be a simple connected graph on $n$ nodes. Then,

$$
E E_{A B C}(G) \geq(n+1) I_{0}(\sqrt{2}),
$$

with equality if and only if $G$ is the path.

Proof Let $G$ be a tree

$$
\begin{aligned}
E E_{A B C}(T) & =\sum_{j=1}^{n} \exp \left(\lambda_{j}\right) \\
& =n+\sum_{j} \lambda_{j}+\sum_{j} \frac{\lambda_{j}^{2}}{2 !}+\cdots \\
& \geq n+2 m=3 n-2
\end{aligned}
$$

It is easy to show that for $n \geq 3$

$$
E E_{A B C}(T) \geq 3 n-2>(n+1) I_{0}(\sqrt{2})=E E_{A B C}\left(P_{n}\right)
$$

Using Corollary 1 we easily see that $E E_{A B C}(G) \geq E E_{A B C}(T) \geq E E_{A B C}\left(P_{n}\right)$, which proves the result.

\section{Summary}

In this work we provide a probabilistic interpretation of the term $\left(k_{i}+k_{j}-2\right) /\left(k_{i} k_{j}\right)$ which appears in the definition of $A B C$ indices. We have shown that it represents the probability of visiting a nearest neighbor edge from one side or the other of a given edge in a graph. Such interpretation in the context of molecular graphs can be related to the polarizing capacity of the bond considered. We then have represented these probabilities in the form of an $A B C$ matrix which has allowed us to consider more general graph-theoretic indices based on the spectral properties of this matrix. For instance, we have studied here the total $A B C$ energy and the Estrada $A B C$ index of the graph as well as the relation between the spectral radius of this matrix and the $A B C$ index. We hope this work opens some new research avenues in the study of the $A B C$ index and properties related to it.

\section{Acknowledgment}

The author thanks the Royal Society for a Wolfson Research Merit Award.

\section{References}

M. Ahmadi, S. Hosseini, P.S. Nowbandegani, On trees with minimal atom bond connectivity index. MATCH-Communications in Mathematical and Computer Chemistry 69, 559-563 (2013a)

M. Ahmadi, S. Hosseini, M. Zarrinderakht, On large trees with minimal atom-bond connectivity index. MATCHCommunications in Mathematical and Computer Chemistry 69(3), 565-569 (2013b)

A. Balaban, O. Ivanciuc, Historical development of topological indices. Topological Indices and Related Descriptors in QSAR and QSPAR, 21-57 (2000)

S.B. Bozkurt, A.D. Güngör, I. Gutman, A.S. Cevik, Randić matrix and Randić energy. MATCH-Communications in Mathematical and Computer Chemistry 64, 239-250 (2010)

J. Chen, X. Guo, Extreme atom-bond connectivity index of graphs. MATCH-Communications in Mathematical and Computer Chemistry 65(3), 713-722 (2011)

J. Chen, J. Liu, X. Guo, Some upper bounds for the atom-bond connectivity index of graphs. Applied Mathematics Letters 25(7), 1077-1081 (2012)

K.C. Das, I. Gutman, B. Furtula, On atom-bond connectivity index. Chemical Physics Letters 511(4), $452-454$ (2011)

H. Deng, S. Radenkovic, I. Gutman, The Estrada index. Applications of Graph Spectra, Math. Inst., Belgrade, 123-140 (2009)

E. Estrada, L. Torres, L. Rodríguez, I. Gutman, An atom-bond connectivity index: modelling the enthalpy of formation of alkanes. Indian Journal of Chemistry. Sect. A: Inorganic, Physical, Theoretical \& Analytical 37(10), 849-855 (1998)

E. Estrada, Characterization of 3D molecular structure. Chemical Physics Letters 319(5), 713-718 (2000)

E. Estrada, Atom-bond connectivity and the energetic of branched alkanes. Chemical Physics Letters 463(4), 422-425 (2008)

E. Estrada, The Structure of Complex Networks: Theory and Applications (Oxford University Press, Oxford, UK, 2012) 
E. Estrada, N. Hatano, Statistical-mechanical approach to subgraph centrality in complex networks. Chemical Physics Letters 439(1), 247-251 (2007)

E. Estrada, D.J. Higham, Network properties revealed through matrix functions. SIAM Review 52(4), 696-714 (2010)

E. Estrada, J.A. Rodriguez-Velazquez, Subgraph centrality in complex networks. Physical Review E 71(5), 056103 (2005)

E. Estrada, N. Hatano, M. Benzi, The physics of communicability in complex networks. Physics Reports 514(3), 89-119 (2012)

G. Fath-Tabar, B. Vaez-Zadeh, A.R. Ashrafi, A. Graovac, Some inequalities for the atom-bond connectivity index of graph operations. Discrete Applied Mathematics 159(13), 1323-1330 (2011)

B. Furtula, A. Graovac, , D. Vukičević, Augmented Zagreb index. Journal of Mathematical Chemistry 48, 370-380 (2010)

B. Furtula, I. Gutman, M. Ivanović, D. Vukičević, Computer search for trees with minimal ABC index. Applied Mathematics and Computation 219(2), 767-772 (2012)

I. Gutman, Degree-based topological indices. Croatica Chemica Acta 86(4), 351-361 (2013)

I. Gutman, B. Furtula, Trees with smallest atom-bond connectivity index. MATCH-Communications in Mathematical and Computer Chemistry 68(1), 131 (2012)

I. Gutman, H. Deng, S. Radenković, The Estrada index: an updated survey. Selected Topics on Applications of Graph Spectra, Math. Inst., Beograd, 155-174 (2011)

I. Gutman, B. Furtula, M. Ivanovic, Notes on trees with minimal atom-bond connectivity index. MATCH-Communications in Mathematical and Computer Chemistry 67(2), 467 (2012a)

I. Gutman, J. Tošović, S. Radenković, S. Marković, On atom-bond connectivity index and its chemical applicability. Indian Journal of Chemistry. Sect. A: Inorganic, Physical, Theoretical \& Analytical 51, 690-694 (2012b)

I. Gutman, B. Furtula, M.B. Ahmadi, S.A. Hosseini, P. Salehi, M.Z. Nowbandegani, The ABC index conundrum. Filomat 27(6), 1075-1083 (2013)

R.A. Horn, C.R. Johnson, Matrix Analysis (Cambridge University Press, Cambridge, UK, 2012)

W. Kutzelnigg, What I like about Hückel theory. Journal of Computational Chemistry 28(1), 25-34 (2007)

X. Li, Y. Shi, A survey on the Randić index. MATCH-Communications in Mathematical and Computer Chemistry 59(1), 127-156 (2008)

X. Li, Y. Shi, I. Gutman, Graph Energy (Springer, New York, USA, 2012)

W. Lin, X. Lin, T. Gao, X. Wu, Proving a conjecture of Gutman concerning trees with minimal ABC index. MATCHCommunications in Mathematical and Computer Chemistry 69(20), 13 (2013)

L. Lovász, Random walks on graphs. Combinatorics, Paul Erdôs is Eighty 2, 1-46 (1993)

M. Randić, Characterization of molecular branching. Journal of the American Chemical Society 97(23), 6609-6615 (1975)

H. Van de Waterbeemd, R. Carter, G. Grassy, H. Kubinyi, Y. Martin, M. Tute, P. Willett, Glossary of terms used in computational drug design (IUPAC recommendations 1997). Pure and Applied Chemistry 69(5), 1137-1152 (1997)

T.S. Vassilev, L.J. Huntington, On the minimum ABC index of chemical trees. Applied Mathematics 2(1), 8-16 (2012)

R. Xing, B. Zhou, F. Dong, On atom-bond connectivity index of connected graphs. Discrete Applied Mathematics 159(15), 1617-1630 (2011) 